\title{
Confidence limits for secondary production estimates: application of the bootstrap to the increment summation method*
}

\author{
T. Brey \\ Alfred Wegener Institut für Polar- und Meeresforschung, Columbusstraße, D-2850 Bremerhaven, FRG \\ Date of final manuscript acceptance: June 1, 1990. Communicated by O. Kinne, Oldendorf/Luhe
}

\begin{abstract}
In field studies, somatic production of animals is often calculated by means of the increment summation method, which is based on consecutive samples from the population. The main disadvantage of this method is the lack of any measurement of variability, therefore the statistical significance of the calculated production value is uncertain. This paper shows that in many cases a nonparametric statistical approach called the "bootstrap" can be used to overcome this problem. By means of this procedure, natural variability of production and production to biomass ratios can be assessed by $95 \%$ confidence intervals, standard deviation or related parameters from a sample of limited size.
\end{abstract}

\section{Introduction}

The somatic production of natural populations of animals is an important parameter both in studies on population dynamics of single species and analysis of energy flow within multi-species ecosystems. In populations with distinct recruitment and separable cohorts the increment summation method (ISM) of Crisp (1984) is often used to calculate production from field data (abundance and mean individual weight of age classes).

Up to now, it has been impossible to estimate the variability of production or production/biomass-ratio $(\mathrm{P} / \mathrm{B})$ within the population. Because there is natural variability in the underlying quantities, abundance, biomass and mean individual weight, this is reflected in the dispersion among replicate samples, and must be described by an adequate parameter. Efron (1979) introduced a nonparametric method known as the "bootstrap" as a tool to solve complicated statistical problems which are intractable by classical methods (Efron 1985, Efron and Gong 1983, Hall 1987). The bootstrap has been applied to several statistical problems in ecology during recent years (Bros and Cowell 1987, Nemec and

* AWI Publication No. 227
Brinkhurst 1988, Smith et al. 1986). This paper describes how the bootstrap can be used to estimate variability of production and related parameters calculated by ISM.

\section{Methods}

Increment summation method (ISM)

This is briefly described here according to Crips (1984). The data required are: $\left(N_{t}\right)$ the abundance of a cohort at time $t ;\left(W_{t}\right)$ the mean individual weight in a cohort at time $t$; where $t=1,2, \ldots n$; and $n=$ number of sampling dates. Production is either estimated from growth increments by

$$
P_{t}=\left(N_{t}+N_{t+1}\right) / 2 \cdot\left(W_{t+1}-W_{t}\right)
$$

or from mortality increments (elimination) by

$$
E_{t}=\left(W_{t}+W_{t+1}\right) / 2 \cdot\left(N_{t}-N_{t+1}\right) .
$$

Total production during a longer period is calculated by adding up the values of $P_{t}$ of consecutive sampling intervals, i.e.

$$
\begin{aligned}
& P=\Sigma P_{t}, \\
& E=\Sigma E_{t} .
\end{aligned}
$$

The production of the whole population is equal to the sum of the production of all cohorts. The mean biomass is calculated by:

$$
B=\Sigma\left(N_{t} \cdot W_{\mathrm{t}}\right) / n,
$$

and the production/biomass-ratio is:

$$
\begin{aligned}
& P / B=\left(\sum P_{t}\right) / B, \\
& E / B=\left(\sum E_{t}\right) / B .
\end{aligned}
$$

ISM gives valid estimates of production, provided that the distance in time between consecutive sampling dates is not too long in relation to growth and mortality rates of the population, and $N_{t}$ and $W_{t}$ are valid estimates of average abundance and individual weight at time $t$ (Cushman et al. 1978, Lapchin and Neveu 1980).

\section{Application of bootstrap to ISM}

In most studies on population dynamics, the sample taken at each sampling date from the population under investigation consists of a number of random sub-samples. These sub-samples are usually pooled to form the sample from which abundance $N_{t}$ and mean 
individual weight $W_{t}$ are calculated. Usually, $N_{t}$ and $W_{t}$ show variability among the sub-samples taken at time $t$, which reflects the variability in the population. We are not able to transform this information on natural dispersion into an estimate of variability of production or related parameters by means of traditional statistical approaches, so this information is lost.

The bootstrap (Efron 1979, 1982, Efron and Gong 1983) is a nonparametric estimation of statistical error of any parameter from a sample of limited size, consisting of independent and identically distributed values. By means of repeated re-sampling of the original sample an empirical probability distribution of the parameter in question is established, from which statistical error can be computed.

Let us assume that we sampled a natural population consisting of one cohort several times during $1 \mathrm{yr}$. At each sampling date we have taken the same number of independent sub-samples of equal sampling-size (i.e., area or volume), which form the sample. Let $\mathbf{X}$ denote the data matrix representing abundance $\left(N_{t s}\right)$ and mean individual weight $\left(W_{t s}\right)$ of one cohort at time $t$ and in subsample $s$ during $1 \mathrm{y}$. Then

$$
\mathbf{X}=\left[x_{t s}\right]=\left[\begin{array}{ll}
x_{11}, & x_{21}, \ldots, x_{n 1} \\
x_{12}, & x_{22}, \ldots, x_{n 2} \\
x_{1 m}, & x_{2 m}, \ldots, x_{n m}
\end{array}\right]
$$

where $x_{t s}=\left(N_{t s}+W_{t s}\right)$ and $N_{t s}=$ no. of animals at time $t$ in sub-sample $s ; W_{t s}=$ mean ind. weight at time $t$ in sub-sample $s ; t=1,2, \ldots, n$; $n=$ number of sampling dates; $s=1,2, \ldots, m ; m=$ number of subsamples; $N_{t}=\Sigma N_{t s} / m ;$ and $W_{t}=\Sigma W_{t s} / m$. This data matrix consists of $n$ columns and $m$ rows, corresponding to the number of samples and sub-samples, respectively. Within each sample, the $m$ sub-samples are assumed to be independent and identically distributed, representing the distribution of $N_{t}$ and $W_{t}$ at time $t$. Subsequent $P_{t}$ and $E_{t}$, however, are not independent, but may be interpreted as a kind of moving average.

A bootstrap data matrix $\mathbf{X}^{*}$ is formed by randomly re-sampling the original data matrix $\mathbf{X}$. Each column of the bootstrap matrix $\mathbf{X}^{*}$ has to be re-sampled from the corresponding column of the original matrix $\mathbf{X}$, i.e. from each column $m$ sub-samples are resampled with replacement to form the $n$ bootstrap samples. From the bootstrap matrix $\mathbf{X}^{*}$ we can calculate mean abundance $N_{t}^{*}$ and mean individual weight $W_{t}^{*}$ at time $t$. The asterisk always denotes data depending on a bootstrap sample. Thus,

$$
\begin{aligned}
& N_{t}^{*}=\Sigma N_{t s}^{*} / m \\
& W_{t}^{*}=\Sigma W_{t s}^{*} / m
\end{aligned}
$$

According to Eqs. (1) to (7) we can now compute $P^{*}, E^{*}, B^{*},(P / B)^{*}$ and $(E / B)^{*}$.

If we re-sample the matrix $\mathbf{X}$ again, we will derive another most likely different - bootstrap matrix $\mathbf{X}^{*}$ and estimates of $P^{*}, E^{*}$, $B^{*},(P / B)^{*}$ and $(E / B)^{*}$, because $N_{t}$ and $W_{t}$ show variability within the samples, i.e. variability between sub-samples. The possible number $\left(s_{b}\right)$ of bootstrap matrices $\mathbf{X}^{*}$, which differ at least in one sub-sample is

$$
s_{b}=\{(2 m-1) ! /[m !(m-1) !]\}^{n}
$$

For example from a matrix with 12 samples and five sub-samples per sample (60 data elements), we can re-sample at most $1.6012 \times 10^{25}$ different bootstrap matrices.

If all these bootstrap matrices were computed, we would obtain the exact probability distribution of $P^{*}$ conditional on the original data matrix $X$, which is due to the sampling variability in the original matrix X. However, in order to get a reasonable estimate of this variability, a random selection of a certain number of bootstrap matrices will be sufficient to get representative empirical probability distributions. These will reflect the impact of the within-sample variability of the basic parameters, $N_{t}$ and $W_{t}$, on dependent parameters like $P^{*}$ and $(P / B)^{*}$. If the data matrix $\mathbf{X}$ is representative for the natural population dynamics (which depends on sampling design) these empirical probability distributions reflect natural variability.
Table 1. Cardium edule. Data set of cockles of age class $1+$, from Kiel Bay, FRG. Ten samples (1 to 10), each consisting of five sub-

\begin{tabular}{|c|c|c|c|c|c|c|c|c|c|c|}
\hline \multirow{2}{*}{$\begin{array}{l}\text { Sam- } \\
\text { ple }\end{array}$} & \multicolumn{2}{|l|}{ I } & \multicolumn{2}{|l|}{ II } & \multicolumn{2}{|l|}{ III } & \multicolumn{2}{|l|}{ IV } & \multicolumn{2}{|l|}{$\mathrm{V}$} \\
\hline & $N_{t s}$ & $W_{t s}$ & $N_{t s}$ & $W_{t s}$ & $N_{t s}$ & $W_{t s}$ & $N_{t s}$ & $W_{t s}$ & $N_{t s}$ & $W_{t s}$ \\
\hline 1 & 14 & 19.20 & 25 & 20.09 & 1 & 1.68 & 2 & 18.06 & 2 & 4.0 \\
\hline 2 & 23 & 23.59 & 4 & 21.07 & 5 & 11.02 & 9 & 15.44 & 29 & 18.77 \\
\hline 3 & 19 & 55.34 & 20 & 59.11 & 20 & 42.95 & 21 & 45.16 & 14 & 57.47 \\
\hline 4 & 19 & 94.07 & 7 & 116.73 & 12 & 93.37 & 19 & 92.16 & 20 & 102.39 \\
\hline 5 & 34 & 95.15 & 33 & 86.70 & 14 & 96.60 & 9 & 85.78 & 11 & 77.52 \\
\hline 6 & 20 & 221.33 & 9 & 221.76 & 2 & 209.10 & 15 & 195.70 & 11 & 230.27 \\
\hline 7 & 1 & 157.30 & 4 & 270.06 & 8 & 247.39 & 3 & 277.42 & 7 & 253.50 \\
\hline 8 & 3 & 251.64 & 4 & 189.13 & 3 & 190.93 & 0 & 0 & 4 & 230.29 \\
\hline 9 & 3 & 196.95 & 1 & 169.72 & 1 & 341.56 & 1 & 189.79 & 1 & 234.25 \\
\hline 10 & 2 & 195.33 & 0 & 0 & 1 & 221.86 & 0 & 0 & 0 & 0 \\
\hline
\end{tabular}
samples (I to V) are given where area of one sub-sample $=0.1 \mathrm{~m}^{2}$. Symbols are: $\left(N_{t s}\right)$ abundance; $\left(W_{t s}\right)$ mean ind. wt in $\mathrm{mg}$ ashfree dry wt

In summary, statistics representing the variability of $P^{*}, E^{*}, B^{*}$, $(P / B)^{*}$ and $(E / B)^{*}$ are calculated according to the following algorithm: (1) Re-sample the original data matrix $\mathbf{X}$ to form the bootstrap matrix $\mathbf{X}^{*}$ by drawing $m$ sub-samples from each sample $t(=1,2, \ldots, n)$ by means of random selection with replacement. (2) Compute mean abundance $N_{t}^{*}$ and mean individual weight $W_{t}^{*}$ at each time $t$ from the bootstrap matrix $\mathrm{X}^{*}$ (Eq. 9). (3) Calculate $P^{*}$, $E^{*}, B^{*},(P / B)^{*}$ and $(E / B)^{*}$. (4) Repeat Step 1 and 2 a large number of times. (5) Estimate the parameters required from the resulting frequency distributions, i.e. the empirical probability distributions of $P^{*}, E^{*},(P / B)^{*}$ and $(E / B)^{*}$ (e.g. average and confidence interval). This procedure can be expanded simply to a data matrix with unequal number of sub-samples per sampling date and also to cases where several age classes are involved.

\section{Testing the bootstrap approach}

In order to test the bootstrap approach, this procedure was applied to artificial data sets and to one natural data set. The artificial data were randomly generated according to the following procedure: Average values of $N_{t}$ and $W_{t}$ for each sample were calculated from linear mortality and growth functions:

$N_{t}=N_{0}-\left(N_{0}-N_{1.0}\right) \cdot t ; \quad N_{0}=190 ; \quad N_{1.0}=10 ; \quad 0 \leqq t \leqq 1.0$

$W_{t}=W_{0}+\left(W_{1.0}-W_{0}\right) \cdot t ; \quad W_{0}=10 ; W_{1.0}=110 ; \quad 0 \leqq t \leqq 1.0$

$P=10000 ; \quad E=10800$.

$N_{t}$ and $W_{t}$ at time $t$ had a negative binomial distribution, as is common in natural populations.

$$
(q-p)^{-k} ; \quad q=1-p ; \quad \text { mean }=k \cdot p ; \quad \text { variance }=k \cdot p \cdot q
$$

The parameter $k$ of the negative binomial distribution was identical for all distributions of $N_{t}$ and $W_{t}$ within one data set, which may be interpreted as a "worst case" situation. The actual values of $N_{t s}$ and $W_{t s}$ were randomly sampled from these distributions.

The natural data set represents the age class $1+$ of the cockle Cardium edule from a station in Kiel Bay (Western Baltic, Brey 1986). It consists of ten samples with five sub-samples each (Table 1). In contrast to Brey (1986), mean individual weight was calculated from length-frequency distributions and length-weight regressions in this study. The mean was selected as the estimator of the average of the resulting distributions, whereas the 2.5 th and 97.5 th percentiles (which approximate the $95 \%$ confidence interval) were taken as estimates of variability (Efron 1982). The skewness of the resulting distributions was estimated by:

Skewness $=3 \cdot($ mean - median $) / S D \quad($ Sachs 1978) 
Table 2. The effect of increasing number of replications $(n)$ on the reproducibility of the distribution of $P^{*}$ (production). Mortality function: $N_{i}=190-(190-10) \cdot t ; 0 \leqq t \leqq 1.0$; growth function: $W_{t}=$ $10+(110-10) \cdot t ; P$ calculated by ISM $=10000$. Distribution of $N$ and $W$ at time $t$ : negative binomial with $k=1.0 ; 12$ samples $(t=0$, $0.08,0.17, \ldots, 1.0)$ and 10 sub-samples/sample; $p[2.5], p[97.5]=2.5$ and 97.5 th percentile of the distribution. Average: arithmetic mean of 10 runs; $\%$ Diff. $=100 \cdot$ (highest value - lowest value)/average

\begin{tabular}{|c|c|c|c|c|c|c|}
\hline \multirow[t]{2}{*}{$n$} & \multicolumn{2}{|c|}{ Mean $P^{*}$} & \multicolumn{2}{|l|}{$p[2.5]$} & \multicolumn{2}{|l|}{$p$ [97.5] } \\
\hline & Average & $\%$ Diff. & Average & $\%$ Diff. & Average & $\%$ Diff. \\
\hline 125 & 10061 & 2.19 & 6132 & 9.05 & 14813 & 8.23 \\
\hline 250 & 9996 & 2.21 & 6083 & 6.79 & 14917 & 4.08 \\
\hline 500 & 10022 & 1.40 & 6095 & 3.62 & 14833 & 5.75 \\
\hline 1000 & 9975 & 1.15 & 6112 & 2.11 & 14774 & 2.20 \\
\hline 2000 & 10013 & 0.47 & 6110 & 2.37 & 14859 & 2.05 \\
\hline
\end{tabular}

Table 3. The effect of increasing dispersion of the within-sample distribution of $N_{t}$ and $W_{t}$ on the distribution of $P^{*}$. Mortality-function: $N_{t}=190-(190-10) \cdot t ; 0 \leqq t \leqq 1.0 ;$ growth-function: $W_{t}=10+$ $(110-10) \cdot t$; distribution of $N_{1}$ and $W_{t}$ at time $t=$ negative binomial distribution with $k=0.66 ; k=0.83 ; k=1.00$. Average values of 5 runs (1000 replications per run), a new data matrix with 8 samples was generated for each run

\begin{tabular}{rrrrrrrr}
\hline$k$ & $p$ & \multicolumn{2}{c}{ Subsamples } & & & & \\
\cline { 3 - 8 } & & 5 & 10 & 20 & 35 & \multicolumn{1}{c}{55} & \multicolumn{1}{c}{80} \\
\hline 0.66 & {$[2.5]$} & 5539 & 6671 & 7783 & 8395 & 8619 & 8852 \\
& {$[97.5]$} & 15314 & 13799 & 12472 & 11811 & 11485 & 11262 \\
0.83 & {$[2.5]$} & 5354 & 6330 & 7369 & 8019 & 8442 & 8711 \\
& {$[97.5]$} & 15816 & 14434 & 12926 & 12102 & 11598 & 11421 \\
1.00 & {$[2.5]$} & 4296 & 6048 & 7227 & 7877 & 8342 & 8580 \\
& {$[97.5]$} & 17356 & 14629 & 13237 & 12412 & 11802 & 11552 \\
\hline
\end{tabular}

Table 4. Distribution of $P^{*}, E^{*},(P / B)^{*}$ and $(E / B)^{*}$ calculated from the data set of Cardium edule (Table 1); one run with 1000 replications; $p[2.5], p[97.5]=2.5$ and 97.5 th percentile of distribution; $95 \%$-limits $=$ mean $\pm 1.96 \cdot$ SD ; skewness $=3 \cdot($ mean - median $) / S D$ ISM $=$ corresponding values computed with mean $N_{t}$ and $W_{t} . P$, $E=\mathrm{gAFDW} \mathrm{m}^{-2} \mathrm{yr}^{-1} ; P / B, E / B=y^{-1}$

\begin{tabular}{lcccccccc}
\hline & Mean & $p[2.5]$ & $p[97.5]$ & $95 \%$-limits & \multicolumn{3}{c}{$\begin{array}{l}\text { Skew- ISM } \\
\text { ness }\end{array}$} \\
\hline$P^{*}$ & 33.3 & 25.7 & 42.2 & 25.3 & 41.3 & 0.01 & 34.7 \\
$E^{*}$ & 33.8 & 26.3 & 42.2 & 25.8 & 41.9 & 0.04 & 35.1 \\
$(P / B)^{*}$ & 3.58 & 3.16 & 4.00 & 3.15 & 4.01 & 0.27 & 3.69 \\
$(E / B)^{*}$ & 3.64 & 3.21 & 4.10 & 3.21 & 4.07 & 0.00 & 3.73 \\
\hline
\end{tabular}

These data sets were used to test: (1) the number of replicated calculations required to get a reasonable estimate of the parameters in question. An artificial data set with 12 samples and 10 sub-samples per sample was used for this experiment. The bootstrap procedure was carried out 10 times each with $125,250,500,1000$ and 2000 replications. The variation of mean, 2.5 th and 97.5 th percentile among the 10 trials with equal number of bootstrap replications was computed. (2) The effect of an increasing size of the data matrix on the distribution of $P^{*}$. Fifty four different matrix sizes were analyzed. The number of samples were $2,4,8,14,22,32,44,58$ and 74 ; the number of sub-samples per sample $5,10,20,35,55$ and 80 . With respect to each combination of sub-samples and samples, the boots- sub-

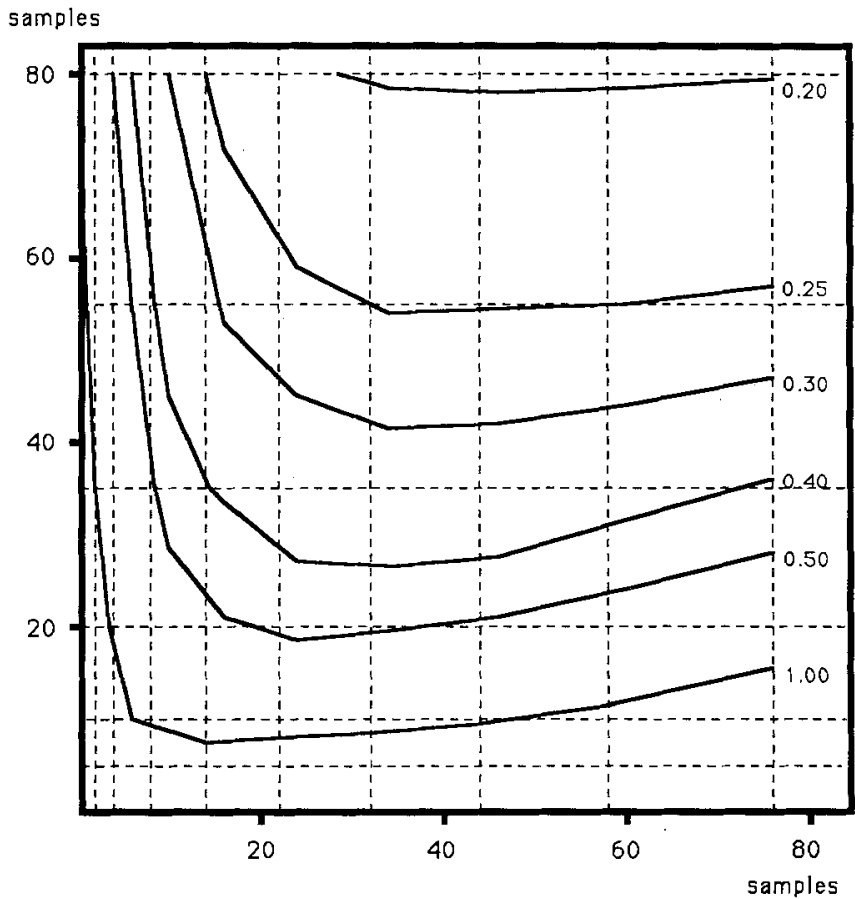

Fig. 1. Effect of an increasing number of samples and subsamples on the $95 \%$ confidence interval of $P^{*}$, if growth and mortality follow linear functions. The plot shows lines of equal confidence interval size (range $=0.20$ to 1.00 ). Confidence interval $(95 \%)=2.5$ to 97.5th percentile, shown here as porportion of mean $P$ (production). $N$ (abundance) and $W$ (weight) at time $t$ are random sampled from negative binomial distributions with $k=1.00$

trap procedure was carried out with five different data matrices and 1000 replications per matrix. (3) The effect of the within-sample distribution of $N_{t}$ and $W_{t}$. The parameter $k\left[=\operatorname{mean}^{2} /\right.$ (variance-mean)] of the negative binomial distribution of $N_{t}$ and $W_{t}$ was changed from 0.66 to 1.00 and the corresponding changes in the distribution of $P^{*}$ were recorded. The data matrix consisted of eight samples and 5 to 80 sub-samples. (4) The Cardium edule data set was used to test the validity of the bootstrap technique as applied to a natural data set characterized by high within-sample variation in abundance and mean weight, and by huge differences in distributions of these parameters among consecutive samples.

\section{Results}

Number of replicated calculations required

Table 2 shows that with an increasing number of replications the variation mean, 2.5 th and 97.5 th percentile decreases among the ten trials. A thousand replications are sufficient to get reproducable probability distributions of $P^{*}$ within $3 \%$ precision.

\section{Effect of an increasing matrix size}

Figure 1 shows the effect of an increasing number of samples and sub-samples on the $95 \%$ confidence interval of $P^{*}$. It is evident from Fig. 1 that the $95 \%$ interval becomes smaller if the number of sub-samples is increased and the number of samples is held constant, i.e. the 2.5 

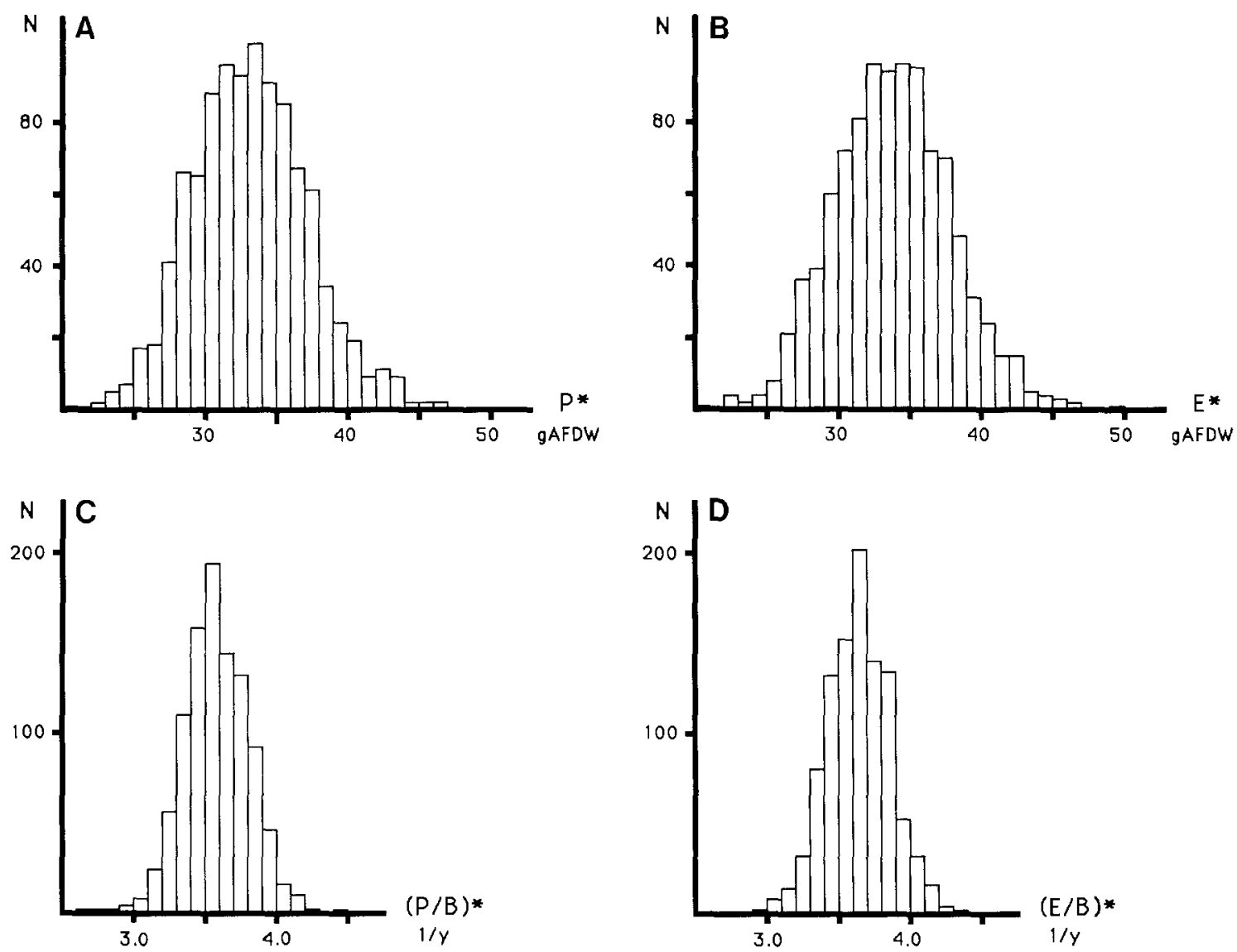

Fig. 2. Cardium edule. Distribution of $(\mathrm{A})$ production $\left(P^{*}\right)$, (B) elimination $\left(E^{*}\right),(\mathrm{C})$ production-biomass ratio $(P / B)$, and $(\mathrm{D})$ eliminationbiomass ratio $\left(E / B^{*}\right)$ with the natural data set of the cockle. One bootstrap trial with 1000 replicates

and 97.5th percentile are shifted towards the mean. If the number of sub-samples is held constant and the number of samples is increased, the $95 \%$ interval decreases sharply first and then increases slowly again.

Effect of the within-sample distribution of $N_{t}$ and $W_{t}$

Table 3 shows the results derived from three data sets which differ only in the variability of $N_{t}$ and $W_{t}$. The $95 \%$ interval of $P^{*}$ increases down each column of Table 3 (increasing $k$ ), i.e. with increasing variability of $N_{t}$ and $W_{t}$.

\section{Natural Cardium edule data set}

In Table 4 , the bootstrap estimates of $P^{*}, E^{*},(P / B)^{*}$ and $(E / B)^{*}$ are compared to the values calculated with mean $N_{t}$ and mean $W_{t}$. The distributions are almost symmetric (Fig. 2) and do not differ significantly from normal distributions $(\alpha=0.05)$. Therefore the parametric 95\%-limits (mean $\pm 1.96 \cdot \mathrm{SD}$ ) are close to the 2.5 and 97.5 th percentiles. Annual production and $P / B$-ratio are estimated to $33.2 \mathrm{~g}$ ashfree dry weight (AFDW) $\mathrm{m}^{-2}( \pm 8.0)$ and 3.58 $( \pm 0.43)$, respectively. The mean values of the bootstrap distributions are somewhat $(2$ to $4 \%$ ) below the ISM estimates. This suggests that the latter may be systematic overestimations of the true average values.

\section{Discussion}

The results presented here do not prove the validity of the application of the bootstrap from a purely mathematical point of view, because the testing of this approach is limited to a few examples. However, my aim is to demonstrate that there may be a simple way to overcome the serious statistical problems related to production calculations. The present results and other potential applications of the bootstrap in production studies will be discussed.

\section{Interpretation of variability estimate}

I will mention the $95 \%$ confidence interval only, because this is a robust nonparametric measure, which is valid for many types of distributions. The $95 \%$ interval of the bootstrap distribution is a dispersion parameter, which depends on both the underlying distributions and the number of data taken from this distribution. Generally we should expect that an increase in the size of the data matrix will lead to an increase in precision of the results. This is indeed the case if the number of sub-samples is increased. An increase in the number of samples, however, does not lead to a continuous decrease in variability, the $95 \%$ range increases again above a certain number of samples (Fig. 1). This pattern results from the formula for production calculation (Eq. 1). The calculation depends 
on the differences in $N_{t}$ and $W_{t}$ - which are mean values per sample - between consecutive samples. The more samples are taken during a period of time, the smaller are the differences in subsequent values of $N_{t}$ and $W_{t}$, and the smaller these differeces are, the more statistical power we need to detect them, i.e. the more sub-samples per sample. Therefore, when the number of samples increases above a certain limit, the variability of the production estimate increases again. These observations have serious implications for sampling strategy (see below).

If the matrix size is constant, an increase in the variability of the underlying data is followed by an increase of the $95 \%$ limits (Table 3), therefore the distribution of $P^{*}$ indeed reflects the natural variability in $N_{t}$ and $W_{t}$.

The estimate of variability presented here does not allow for quantitative information on the real spatial dispersion of production in the natural population under investigation, because the field samples are taken at random from this population. We do not know the spatial relationships between sub-samples taken at subsequent sampling dates. However, the estimate of variability is an expression of variation in space, which may be due to stationary animals under spacially different conditions or to migrating animals. If sampling design is comparable, the bootstrap estimates of variability could also be used to compare directly the spatial component of dispersion in production among different populations, as shown in Table 2.

\section{Existence of a central limit theorem}

One interesting result of the present study is that the resulting distributions of $P^{*}, E^{*}, B^{*},(P / B)^{*}$ and $(E / B)^{*}$ seem to tend towards normality with increasing matrix size, independently of the type of the underlying withinsample distributions of $N_{t}$ and $W_{t}$ (see Figs. 2 and 3). If this assumption is verified by a more extensive investigation, it suggests the existence of a central limit theorem for the bootstrap application on the ISM. A central limit theorem states that the distribution of a parameter derived by random selection from various distributions tends towards normality with an increasing number of underlying distributions. Some central limit theorems are valid for moving average processes (Anderson 1971), which are to some extent related to the ISM procedure. However, no single theorem corresponds exactly to the present situation, where the parameter in question is a function of two random variables drawn from non-stochastic, unknown distributions.

\section{Implications for sampling strategy}

The results of the present study have also important implications on the sampling strategy in field studies on production. Fig. 1 shows that in order to get a 95\% confidence of mean $P^{*}$ interval which is, let us say, smaller than $0.5 \cdot$ mean $P^{*}$, one can take either 5 samples with 40 sub-samples each (=230 sub-samples), or 20 samples with 19 sub-samples each (=380 sub-samples). It is evi-

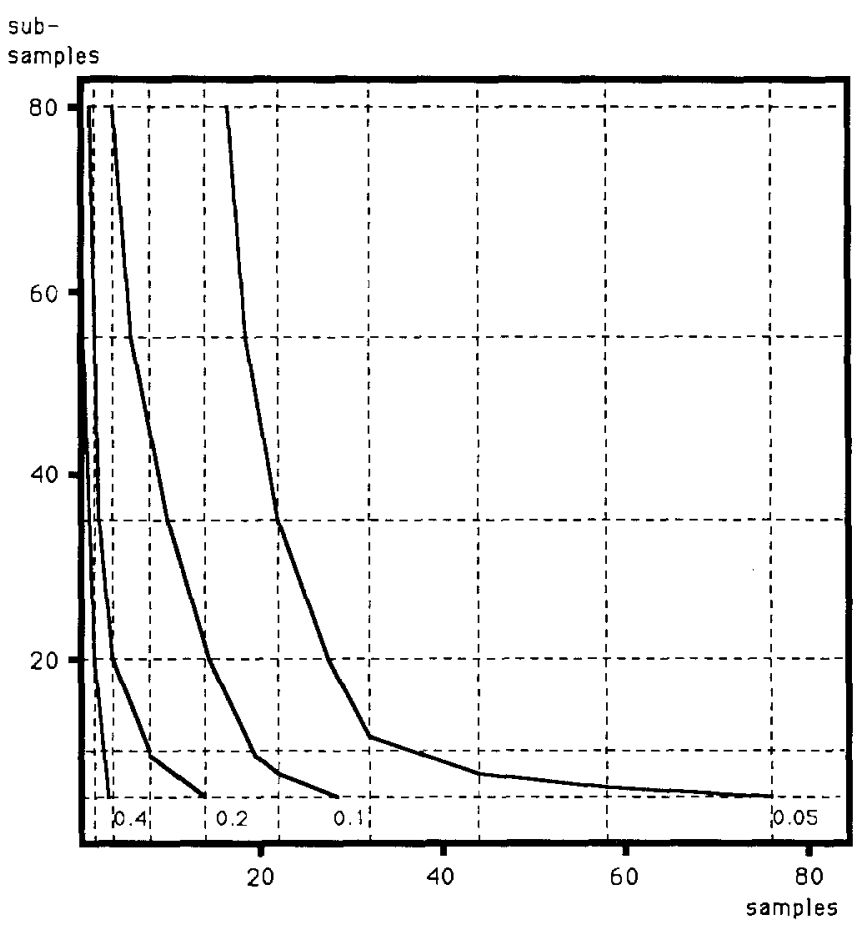

Fig. 3. Effect of an increasing number of samples and subsamples on the skewness of the distribution of $P^{*}$ (production). The plot shows lines of equal skewness (range $=0.05$ to 0.4 ). Skewness $=3 \cdot($ mean - median) $/ \mathrm{SD} . N$ (abundance) and $W$ (weight) at time $t$ are random sampled from negative binomial distributions with $k=1.00$

dent from these values that the number of sub-samples should be increased preferably in order to get a more precise estimate of production. However, the data shown in Fig. 1 depend on linear growth and mortality functions. In most natural populations, growth and mortality follow nonlinear functions, but ISM is always a linear interpolation between two consecutive samples. Therefore, reducing the number of samples results in increasing a systematic error in the production calculations. In order to overcome this problem, the time interval between consecutive samples should be related to the rates of change in $N_{t}$ and $W_{t}$ over time. The distance may be larger for populations with lower growth and mortality rates and smaller for populations with higher rates. Within the investigation of a single age-class, unequal time steps between samples may be the most efficient strategy. The step should be small if $N_{t}$ and $W_{t}$ change fast - usually in the first part of a cohort's life - and become larger with decreasing changes in these parameters. However, in populations consisting of several age-classes the sampling strategy has to be a compromise.

\section{Limitations, advantages and potential applications}

The only serious limitation of the bootstrap is the number of sub-samples per sample. With respect to the assymptotic efficiency of the bootstrap which is not discussed here, i.e. the relation between the number of data and the statistical power, this number should not be to small. It is 
always more efficient to take many small samples than few large ones.

The application of the bootstrap to production studies has at least two advantages: (1) information about natural variability, which is hidden in the data, can be expressed as an estimate of variation (standard deviation or confidence limits) without additional sampling effort; (2) differences in the production of different populations or during different years can be tested statistically.

Not only the ISM but all techniques of production calculation applied to data sets consisting of several random sub-samples per sample may be improved by the bootstrap e.g. the size-frequency method (Hynes and Coleman 1968, Menzie 1980) or methods based on weight-specific growth rates (see Crisp 1984).

Acknowledgements. I thank B. Efron and M. Martin, both from Stanford University, whose critical revision improved the manuscript substantially.

\section{Literature cited}

Anderson, T. W. (1971), The statistical analysis of time series. Wiley, New York

Brey, T. (1986). Estimation of annual P/B-ratio and production of marine benthic invertebrates from length-frequency data. Ophelia (Suppl.) 4: 45-54

Bros, W. E., Cowell, B. C. (1987). A technique for optimizing sample size (replication). J. exp. mar. Biol. Ecol. 114: 63-71
Crisp, D. J. (1984). Energy flow measurements. In: Holme, N. A., McIntyre, A. D. (eds.) Methods for the study of marine benthos, 2nd edn. Blackwell, Oxford, London

Cushman, R. M., Shugart, H. H., Hildebrand, S. G., Elwood, J. W. (1978). The effect of growth curve and sampling regime on instantaneous-growth, removal-summation, and Hynes/Hamilton estimates of aquatic insect production: a computer simulation. Limnol. Oceanogr. 23: 184-189

Efron, B. (1979). Bootstrap methods: another look at the jackknife. Ann. Statist. 7: 1-26

Efron, B. (1982). The jackknife, the bootstrap, and other resampling plans. Society for Industrial and Applied Mathematics, Monograph No. 38, Philadelphia

Efron, B. (1985). Bootstrap confidence intervals for a class of parametric problems. Biometrika 72: 45-58

Efron, B., Gong, G. (1983). A leisurely look at the bootstrap, the jack-knife, and cross-validation. Am. Statist. 37: 36-48

Hall, P. (1987). On the bootstrap and likelihood-based confidence regions. Biometrika 74: $481-493$

Hynes, H. B. N., Coleman, M. J. (1968). A simple method of assessing the annual production of stream benthos. Limnol. Oceanogr. 13: $569-573$

Lapchin, L., Neveu, A. (1980). The production of benthic invertebrates: comparison of different methods. Acta Oecologica 1: 307-322

Menzie, C. A. (1980). A note on the Hynes method of estimating secondary production. Limnol. Oceanogr. 25: 770-773

Nemec, A. F. L., Brinkhurst, R. O. (1988). Using the bootstrap to assess statistical significance in the cluster analysis of species abundance data. Can. J. Fish. aquat. Sciences 45: 965 970

Sachs, L. (1978). Angewandte Statistik, 4th edn. Springer, Berlin, New York

Smith, E. P., Genter, R. B., Cairns, J. (1986). Confidence intervals for the similarity between algal communities. Hydrobiologia 139: $237-245$ 